\title{
Plasma vascular endothelial growth factor, angiopoietin-2, and soluble angiopoietin receptor tie-2 in diabetic retinopathy: effects of laser photocoagulation and angiotensin receptor blockade
}

\author{
P L Lip, S Chatteriee, G J Caine, M Hope-Ross, J Gibson, A D Blann, G Y H Lip
}

Br J Ophthalmol 2004;88:1543-1546. doi: 10.1136/bjo.2004.048587

Background: Proliferative diabetic retinopathy (PDR) may be a response to abnormal angiogenic growth factors such as vascular endothelial growth factor (VEGF), angiopoietin-2 (Ang-2), and the soluble angiopoietin receptor tie-2. The authors hypothesised the following: (a) there are differences in plasma levels of these growth factors in different grades of diabetic retinopathy; and (b) that the effects of intervention with panretinal laser photocoagulation (PRP) for PDR, and angiotensin receptor blockade (using eprosartan) for patients with other grades of diabetic retinopathy will be to reduce levels of the growth factors.

Methods: Cross sectional and interventional study (using PRP and eprosartan) in diabetic patients. VEGF, Ang-2, and tie-2 were measured by ELISA.

Results: VEGF $(p<0.001)$ and Ang-2 levels $(p<0.001)$ were significantly higher in 93 diabetic patients compared to 20 healthy controls, with the highest levels in grade 2 and grade 3 diabetic retinopathy $(p<0.05)$. Tie- 2 was lower in diabetics compared to controls $(p=0.008)$, with no significant differences between the diabetic subgroups. Overall, VEGF significantly correlated with Ang-2 $(p<0.001)$ and tie-2 $(p=0.004)$ but the correlation between Ang-2 and tie-2 levels was not significant $(p=0.065)$. Among diabetic patients only, VEGF levels were significantly correlated with Ang-2 $(p<0.001)$ and tie-2 $(p<0.001)$; the correlation between Ang-2 and tie-2 levels was also significant $(p<0.001)$. There were no statistically significant effects of laser photocoagulation on plasma VEGF, Ang-2, and tie- 2 in the 19 patients with PDR, or any effects of eprosartan in the 28 patients with non-proliferative diabetic retinopathy.

Conclusion: Increased plasma levels of VEGF and Ang-2, as well as lower soluble tie-2, were found in diabetic patients. The highest VEGF and Ang-2 levels were seen among patients with pre-proliferative and proliferative retinopathy, but there was no relation of tie-2 to the severity of retinopathy. As the majority of previous research into Ang2 and tie-2 has been in relation to angiogenesis and malignancy, the present study would suggest that Ang-2 and tie- 2 may be used as potential indices of angiogenesis in diabetes mellitus (in addition to VEGF) and may help elucidate the role of the angiopoietin/tie-2 system in this condition.

S

evere visual impairment associated with diabetic retinopathy is primarily a result of diabetic maculopathy or a sequel of retinal neovascularisation, leading to vitreous haemorrhage and/or tractional retinal detachment. In diabetic patients, some of the earliest pathophysiological retinovascular changes include selective loss of capillary pericytes, impairment of retinal vascular autoregulation, and the failure of the capillary circulation. ${ }^{1}$ These changes subsequently lead to increased retinal vascular permeability, chronic retinal hypoxia and extensive retinal ischaemia, eventually resulting in retinal neovascularisation. These processes are thought to be mediated by various growth factors, such as vascular endothelial growth factor (VEGF). ${ }^{2}{ }^{3}$

More recently, oncology research has identified other growth factors, such as those of the angiopoietin family, that appear to have a major role in angiogenesis and metastasis. ${ }^{4}$ The roles of two members of the angiopoietin family, angiopoietin- 1 and angiopoietin-2 (Ang-1 and Ang-2) are becoming increasingly understood, and may act alongside VEGF in tumour angiogenesis, as well as in retinal neovascularisation. ${ }^{6}$ Indeed, VEGF has been shown to be responsible for the initiation of angiogenesis through recruitment and proliferation of endothelial cells, a process that Ang-1 potentiates..$^{78}$ The angiopoietins interact with the endothelium via endothelial cell specific tyrosine kinase receptors, tie- 1 and tie- 2 . The role of the tie- 2 receptor has been examined in neovascularisation associated with malignancy, and soluble tie-2 has been detected in the plasma of patients with vascular disease, ${ }^{9}$ but we are not aware of previous data on plasma Ang-2 and tie-2 levels in patients with diabetes, and the relation with diabetic retinopathy.

Pan retinal laser photocoagulation is the current management for proliferative diabetic retinopathy, although the possibility that drug treatment (for example, with drugs that block the renin-angiotensin system) is an attractive option. In non-hypertensive patients aged 20-59 with little or no nephropathy and with type 1 diabetic, a recent 2 year randomised double blind placebo controlled trial using retinal photographs at baseline and follow up (24 months) reported a reduction in the progression of retinopathy, as well as less development of proliferative retinopathy, in patients treated with the angiotensin converting enzyme (ACE) inhibitor, lisinopril. ${ }^{10}$

VEGF is a potent angiogenic growth factor. Raised plasma levels of this growth factor in patients with cancer may relate to ongoing angiogenesis. ${ }^{11}$ In the eye, numerous types of retinal cells are recognised to produce VEGF, including retinal pigment epithelial cells, pericytes, endothelial cells, Muller cells, and astrocytes. ${ }^{12}{ }^{13}$ Intraocular VEGF levels have

Abbreviations: $\mathrm{ACE}$, angiotensin converting enzyme; Ang-2, angiopoietin-2; IQR, interquartile range; PDR, proliferative diabetic retinopathy; PRP, panretinal laser photocoagulation; VEGF, vascular endothelial growth factor 
also been studied in animal models and human vitreous fluid, where the levels are found to be high in patients with active intraocular neovascularisation, such as proliferative diabetic retinopathy, ischaemic central retinal vein occlusion, rubeosis iridis, and retinopathy of prematurity. ${ }^{12}$ We recently reported a small pilot study of 18 patients with proliferative retinopathy (secondary both to diabetes and retinal vein occlusion), who had significantly raised plasma VEGF levels when compared to diabetics with background retinopathy only and healthy controls. ${ }^{14}$ Following panretinal laser photocoagulation of the patients with proliferative retinopathy, there was a significant reduction in plasma VEGF levels at 4 months follow up.

The aim of this study was to extend our previous pilot data in patients with proliferative retinopathy, with the following: (a) a cross sectional study in diabetic patients only, examining the relation of VEGF, Ang-2, and the soluble angiopoietin receptor tie-2 to different grades of diabetic retinopathy; and (b) to assess the effects of intervention with panretinal laser photocoagulation (PRP) for proliferative diabetic retinopathy, and angiotensin receptor blockade (using eprosartan) for patients with other grades of diabetic retinopathy.

\section{PATIENTS AND METHODS}

Patients with proliferative retinopathy secondary to diabetes with no previous PRP therapy were recruited from the medical retina and diabetic clinics. Patients with type 1 diabetes with pre-proliferative retinopathy and background diabetic retinopathy were recruited if their resting diastolic blood pressure was $>80 \mathrm{~mm} \mathrm{Hg}$ and/or systolic blood pressure $>130 \mathrm{~mm} \mathrm{Hg}$. Patients with type 2 diabetes with background diabetic retinopathy who had not received previous laser therapy were also studied. The diagnosis of proliferative retinopathy was made on slit lamp biomicroscopy with the aid of fluorescein angiography in doubtful cases. Trained photographers took fundus photographs of two $50^{\circ}$ fields of each eye centred on the macula temporal and disc nasal. The diabetic retinopathy was graded according to the worse affected eye by masked expert observers using retinal photographs. Based on the modified Airlie House photographic classification, retinopathy was graded as grade 1 or non-proliferative retinopathy, grade 2 or preproliferative retinopathy, and grade 3 or proliferative retinopathy. ${ }^{15}$

Twenty eight patients with proliferative retinopathy underwent PRP, and were re-examined at 2 months. Nineteen patients with pre-proliferative retinopathy, and background diabetic retinopathy, who are not taking an ACE inhibitor or angiotensin receptor antagonist at baseline, were treated with eprosartan $600 \mathrm{mg}$ daily, and the dose increased over 2 weeks to $800 \mathrm{mg}$, if diastolic blood pressure was more than
$80 \mathrm{~mm} \mathrm{Hg}$ at a $\mathrm{l}$ week visit. Patients were also re-examined and had repeat blood samples for research indices at 2 months.

Exclusion criteria for all subjects were a history of renal or liver impairment, malignancy, cardiovascular disease (previous myocardial infarction, stroke, peripheral artery disease), uncontrolled hypertension (>160/95 mm Hg), connective tissue disease, deep vein thrombosis, or pulmonary embolism (confirmed, where appropriate, by laboratory tests). Baseline blood samples were compared to healthy control subjects recruited from those attending hospital for non-acute minor surgical conditions, such as cataract surgery and hernia surgery, and from members of the hospital staff. None of the patients or healthy controls had a history of renal or liver disease, malignancy, connective tissue disease, deep vein thrombosis, or pulmonary embolism. The project had the approval of the ethics committee of West Birmingham Health Authority and all patients gave informed consent to the study.

A $20 \mathrm{ml}$ blood sample was taken at baseline and in those undergoing with intervention with PRP or eprosartan, at 2 months. VEGF, tie-2, and Ang-2 were measured by ELISA using commercial reagents. ${ }^{9}$

\section{Power calculations and statistical analysis}

Based on our pilot study, ${ }^{13}$ we calculated that 18 patients and 18 controls would have $90 \%$ power at the $\mathrm{p}<0.01$ significance level to detect a change of one standard deviation in VEGF levels. As growth factor data are non-parametrically distributed, it is presented as median (IQR, interquartile range). Differences between patients and healthy controls were compared using the one way analysis of variance (ANOVA) or Kruskal-Wallis test as appropriate, while paired comparisons between levels at baseline and 4 months post-laser/ drug were compared using the paired Wilcoxon test. Data were correlated using Spearman's rank method. Data were entered onto a computerised database and statistical calculations performed on a microcomputer using a commercially available statistical package (Minitab version 12 for Windows, Minitab Inc, State College, PA, USA). A value of $\mathrm{p}<0.05$ was considered significant in all statistical analyses.

\section{RESULTS}

Plasma VEGF levels $(\mathrm{p}<0.001)$ and Ang-2 levels $(\mathrm{p}<0.001)$ were significantly higher in diabetic patients compared to controls, with the highest median levels in grade 2 and grade 3 diabetic retinopathy (Tukey's post hoc test after log transformation, $\mathrm{p}<0.05$ ) (table 1 ). Levels of tie- 2 were lower in diabetics compared to controls $(p=0.008)$, with no significant differences between the diabetic subgroups (Tukey's post hoc test after log transformation, $\mathrm{p}>0.05$ ) (table 1).

Table 1 Plasma vascular endothelial growth factor, angiopoietin-2, and soluble angiopoietin receptor tie-2 in diabetic retinopathy

\begin{tabular}{|c|c|c|c|c|c|}
\hline & \multirow[b]{2}{*}{ Healthy controls } & \multicolumn{3}{|c|}{ Diabetic retinopathy } & \multirow[b]{2}{*}{ p Value } \\
\hline & & Grade 1 & Grade 2 & Grade 3 & \\
\hline Number & 20 & 38 & 22 & 33 & \\
\hline Males (n) & 12 & 25 & 11 & 25 & \\
\hline Age (years) & 54.5 (15.3) & $61.6(14.7)$ & $61.6(14.1)$ & $61.3(13.8)$ & 0.275 \\
\hline VEGF (pg/ml) & $29(25-58)$ & $110(68-170)$ & 140 (120-208) & $140(60-275)$ & $<0.001$ \\
\hline Ang-2 (ng/ml) & $1.2(0.75-1.75)$ & $1.7(1.4-2.3)$ & $2.4(1.6-5.1)$ & $2.3(1.6-4.0)$ & $<0.001$ \\
\hline tie-2 (ng/ml) & $7.8(6.1-9.3)$ & $3.0(2.0-5.4)$ & $4.0(2.5-8.2)$ & $4.0(2.0-10.0)$ & 0.008 \\
\hline
\end{tabular}

Data presented as median and IQR, comparisons using the Kruskal-Wallis test.

Grade 1, non-proliferative retinopathy; grade 2, preproliferative retinopathy; grade 3, proliferative retinopathy.

VEGF, vascular endothelial growth factor; Ang-2, angiopoietin-2; tie-2, soluble angiopoietin receptor tie-2. 
Table 2 Effects of laser photocoagulation in 19 patients with proliferative diabetic retinopathy

\begin{tabular}{llll}
\hline & Baseline & 2 months & p Value \\
\hline VEGF $(\mathrm{pg} / \mathrm{ml})$ & $140(70-160)$ & $130(100-170)$ & 0.647 \\
Ang-2 (ng/ml) & $1.9(1.6-2.7)$ & $1.8(1.5-2.5)$ & 0.301 \\
tie-2 (ng/ml) & $3.0(1.5-6.5)$ & $2.5(1.75-5.0)$ & 0.850 \\
\hline VEGF, vascular endothelial growth factor; Ang-2, angiopoietin-2; tie-2, soluble angiopoietin receptor tie-2.
\end{tabular}

\section{Correlations}

In the patients and controls, VEGF levels were significantly correlated with Ang- $2 \quad(r=0.587, \mathrm{p}<0.001)$ and tie-2 $(r=0.27, \mathrm{p}=0.004)$. However, the correlation between Ang-2 and tie-2 levels was not significant $(r=0.178$, $\mathrm{p}=0.065)$. Age only significantly correlated with Ang-2 levels $(r=0.234, \mathrm{p}=0.013)$. Among diabetic patients only, VEGF levels were significantly correlated with Ang-2 $(r=0.442, \mathrm{p}<0.001)$ and tie-2 $(r=0.518, \mathrm{p}<0.001)$. The correlation between Ang-2 and tie-2 levels was significant $(r=0.509, \mathrm{p}<0.001)$. There were no correlations with age among the diabetic subgroup.

\section{Effects of intervention}

There were no statistically significant effects of laser photocoagulation on plasma VEGF, Ang-2, and tie-2 levels in the 19 patients with proliferative diabetic retinopathy (table 2). Similarly, there were no statistically significant effects of eprosartan in the 28 patients with non-proliferative diabetic retinopathy (table 3 ).

\section{DISCUSSION}

In this study, we have confirmed previous observations of increased plasma VEGF levels in diabetic patients, as well as previous observations of higher plasma VEGF levels in patients with more severe retinopathy, ${ }^{13}$ with the highest median VEGF levels among patients with pre-proliferative and proliferative retinopathy (grades 2 and 3). We also show, for the first time, that Ang-2 levels are increased in diabetics, with the highest levels among patients with grade 2 and 3 retinopathy. Furthermore, we also demonstrate that soluble tie-2 levels are lower among diabetics than controls, with no relation to the severity of retinopathy.

VEGF correlated with both Ang-2 and tie-2 among diabetics and in the whole study cohort, in keeping with these three indices being possible indices of angiogenesis. Interestingly, the correlation between Ang-2 and tie-2 was only significant in the diabetic patients, as has previously been shown in cancer patients. ${ }^{10}$ We have previously reported plasma tie-2 levels to be higher in patients with coronary artery disease compared to healthy controls, as well as a significant correlation between tie-2 and VEGF in this patient group (Spearman $r=0.59, \mathrm{p}<0.001$ ). ${ }^{15}$ That levels of tie- 2 correlate with other angiogenic markers is consistent with work suggesting interactions between the VEGF and angiopoietin families of growth factors and receptors. ${ }^{10}$ Certainly, Ang-2 appears to be an antagonist to tie-2, and in tumour models, high expression of Ang-2 coincides with vessel regression and high levels of VEGF at the tumour margin-in keeping with robust angiogenesis. ${ }^{16}$ Indeed, Ang-2 may cause destabilisation of the vascular network, promoting the greater expression of VEGF.

In contrast with our previous pilot study, ${ }^{14}$ there was no significant reduction in VEGF levels after panretinal photocoagulation in the present study. Our previous pilot study included patients with proliferative retinopathy secondary to both diabetes and retinal vein occlusion, while the present study was confined to diabetic patients only and significant cardiovascular disease or hypertension were exclusion criteria. Many patients in the present cross sectional study were also treated with ACE inhibitors at baseline (reflecting current clinical practice), and pre-intervention VEGF levels were also much lower that than reported in our pilot study, making a substantial change secondary to intervention more difficult to show. These reasons may also account for the corresponding lack of effect of panretinal photocoagulation on Ang-2 and tie-2 levels.

Similarly, we failed to show a significant effect of eprosartan on our research indices in patients with preproliferative diabetic retinopathy. In our previous study in hypertensive patients (a minority of whom had concomitant diabetes), treatment with either an ACE inhibitor or losartan resulted in a significant reduction in plasma VEGF levels, ${ }^{17}$ although we are not aware of any previous published data on the effect of angiotensin receptor blockers on Ang-2 or tie-2 in diabetes.

This study is limited by its cross sectional design and numbers in the intervention arms are relatively small. It is possible that patients with diabetes had significant underlying atherosclerotic vascular disease, although we attempted to exclude such patients to minimise confounding effects.

In conclusion, we have shown increased plasma levels of VEGF and Ang-2, as well as lower soluble tie-2, in diabetic patients. The highest median VEGF and Ang-2 levels were seen among patients with pre-proliferative and proliferative retinopathy, but there was no relation of tie- 2 to the severity of retinopathy. As the majority of previous research into Ang2 and tie-2 has been in relation to angiogenesis in malignancy, and the present study would suggest that Ang2 and tie- 2 may be used as a potential indices of angiogenesis in diabetes mellitus (in addition to VEGF) and may help elucidate the role of the angiopoietin/tie-2 system in this condition.

\section{ACKNOWLEDGEMENTS}

We acknowledge the support of the Sandwell and West Birmingham Hospitals NHS Trust Research and Development Programme for the

Table 3 Effects of eprosartan in 28 patients with non-proliferative diabetic retinopathy

\begin{tabular}{llll}
\hline & Baseline & 2 months & p Value \\
\hline VEGF $(\mathrm{pg} / \mathrm{ml})$ & $135(60-165)$ & $125(70-220)$ & 0.367 \\
Ang-2 $(\mathrm{ng} / \mathrm{ml})$ & $2.0(1.4-5.1)$ & $2.0(1.4-13.8)$ & 0.899 \\
tie-2 $(\mathrm{ng} / \mathrm{ml})$ & $3.5(1.8-11.0)$ & $5.0(2.5-14.3)$ & 0.584 \\
\hline
\end{tabular}

Paired Wilcoxon test, all $\mathrm{p}=$ NS. Data presented as median and interquartile range.

VEGF, vascular endothelial growth factor; Ang-2, angiopoietin-2; tie-2, soluble angiopoietin receptor tie- 2 . 
Haemostasis, Thrombosis and Vascular Biology Unit. This project is supported by the Royal Blind Asylum and School/Scottish National Institute for the War Blinded Award of the Royal College of Surgeons of Edinburgh, as well as an educational non-promotional grant from Solvay Healthcare Limited, which also provided the eprosartan.

\section{Authors' affiliations}

P L Lip, S Chatteriee, G J Caine, A D Blann, G Y H Lip, Haemostasis, Thrombosis and Vascular Biology Unit, University Department of Medicine, City Hospital, Birmingham, UK

P L Lip, M Hope-Ross, J Gibson, Birmingham and Midland Eye Centre, City Hospital, Birmingham, UK

Correspondence to: Professor G Y H Lip, University Department of Medicine, City Hospital, Birmingham B18 7QH, UK; g.y.h.lip@bham.ac. uk

Accepted for publication 31 May 2004

\section{REFERENCES}

1 Witmer AN, Vrensen GF, Van Noorden CJ, et al. Vascular endothelial growth factors and angiogenesis in eye disease. Prog Retin Eye Res 2003;22:1-29.

2 Caldwell RB, Bartoli M, Behzadian MA, et al. Vascular endothelial growth factor and diabetic retinopathy: pathophysiological mechanisms and treatment perspectives. Diabetes Metab Res Rev 2003;19:442-55.

3 Lu M, Adamis AP. Vascular endothelial growth factor gene regulation and action in diabetic retinopathy. Ophthalmol Clin North Am 2002;15:69-79.

4 Lauren J, Gunji Y, Alitalo K. Is angiopoietin-2 necessary for the initiation of tumor angiogenesis? Am J Pathol 1998;153:1333-9.

5 Metheny-Barlow LJ, Li LY. The enigmatic role of angiopoietin-1 in tumor angiogenesis. Cell Res 2003;13:309-17.
6 Takagi $\mathbf{H}$, Koyama S, Seike $\mathrm{H}$, et al. Potential role of the angiopoietin/tie2 system in ischemia-induced retinal neovascularization. Invest Ophthalmol Vis Sci 2003;44:393-402.

7 Holash J, Wiegand SJ, Yancopoulos GD. New model of tumor angiogenesis: dynamic balance between vessel regression and growth mediated by angiopoietins and VEGF. Oncogene 1999;18:5356-62.

8 Holash J, Maisonpierre PC, Compton D, et al. Vessel cooption, regression, and growth in tumors mediated by angiopoietins and VEGF. Science 1999;284:1994-8.

9 Chung NA, Makin AJ, Lip GY. Measurement of the soluble angiopoietin receptor tie-2 in patients with coronary artery disease: development and application of an immunoassay. Eur J Clin Invest 2003;33:529-35.

10 Chaturvedi N, Sjolie AK, Stephenson JM, et al. Effect of lisinopril on progression of retinopathy in normotensive people with type 1 diabetes. The EUCLID Study Group. EURODIAB Controlled Trial of Lisinopril in InsulinDependent Diabetes Mellitus. Lancet 1998;351:28-31.

11 Caine GJ, Blann AD, Stonelake PS, et al. Plasma angiopoietin-1, angiopoietin-2 and Tie-2 in breast and prostate cancer: a comparison with VEGF and Flt-1. Eur J Clin Invest 2003:33:883-90.

12 Aeillo LP, Avery RL, Arigg PG, et al. Vascular endothelial growth factor in ocular fluids of patients with diabetic retinopathy and other retinal disorders. N Engl J Med 1994;331:1480-7.

13 Yang $Q$, Zwijsen $A$, Slegers $H$, et al. Purification and characterisation of VEGF/VPF secreted by human retinal pigment epithelial cells. Endothelium 1994;2:73-85.

14 Lip PL, Belgore F, Blann AD, et al. Plasma VEGF and soluble VEGF receptor Flt- 1 in proliferative retinopathy: relationship to endothelial dysfunction and laser treatment. Invest Ophthalmol Vis Sci 2000:41:21 15-19.

15 Sjolie AK, Stephenson J, Aldington S, et al. Retinopathy and vision loss in insulin-dependent diabetes in Europe. The EURODIAB IDDM complications study. Ophthalmology 1997; 104:252-60.

16 Zhang L, Yang N, Park JW, et al. Tumor-derived vascular endothelial growth factor up-regulates angiopoietin-2 in host endothelium and destabilizes host vasculature, supporting angiogenesis in ovarian cancer. Cancer Res 2003;63:3403-12.

17 Belgore FM, Blann AD, Li-Saw-Hee FL, et al. Plasma levels of vascular endothelial growth factor and its soluble receptor (SFlt-1) in essential hypertension. Am J Cardiol 2001;87:805-7, A9. 